\title{
Open door expansive laminoplasty and postoperative axial symptoms: a comparative study between two different procedures
}

Authors Yu Sun, Fengshan Zhang, Shaobo Wang, Li Zhang, Shengfa Pan, Miao Yu, Sujun Qiu Institution Department of Orthopaedic Surgery, Institute of Spine Surgery, Peking University Third Hospital, Beijing, China

\begin{tabular}{l|l|}
\hline Final class of & Yes \\
evidence-treatment & \\
\hline Study design: & \\
\hline RCT & \\
\hline Cohort & \\
\hline Case control & \\
\hline Case series & \\
\hline Methods & \\
\hline Concealed allocation (RCT) & \\
\hline Intention to treat (RCT) & \\
\hline Blinded/independent & \\
evaluation of primary outcome & \\
\hline Follow-up $\geq 85 \%$ & \\
\hline Adequate sample size & \\
\hline Control for confounding & \\
\hline Overall class of evidence & III \\
\hline
\end{tabular}

\section{ABSTRACT}

Study design: Retrospective cohort

Objective: To determine if there are differences in important clinical outcomes between patients treated with a "preservation of the unilateral posterior muscular-ligament complex (PMLC) procedure" and a "hinge side anchoring procedure (HSAP)" for the treatment of cervical spondylotic myelopathy (CSM).

Methods: Nine hundred and forty-six patients underwent the above procedures for treatment of CSM between January 2006 and December 2009. Five hundred and fifty-two (58\%) subjects met study criteria and 136 were analyzed (76\% unavailable for follow-up). One group (70 patients) received a "preservation of unilateral PMLC procedure" and another group (66 patients) received an HSAP. The rate and severity of postoperative axial symptoms were assessed, and the changes of cervical-spine curvature and postoperative decompression were measured.

Results: The mean time to follow-up in the preservation of unilateral PMLC group was 6.9 months (range, 6-8 months) and the HSAP group was 6.4 months (6-8 months). The overall rate of any axial symptoms (AS) among all subjects was $35 \%$. No AS was reported in $45.7 \%$ of the preservation of unilateral PMLC group and $23.8 \%$ of the HSAP group $(P=.008)$. Severe AS was reported in $4.3 \%$ and $11.1 \%$ of patients per group, respectively $(P=.14)$, (Table 2). Cervical spinal lordosis was improved in the unilateral PMLC group and decreased in the HSAP group. The risk of losing lordosis was more than two times higher in the HSAP group compared with the unilateral PMLC group $(70 \%$ and $34 \%$, respectively; $P<.001)$.

No financial support was received for this study. IRB (or equivalent) approval. 
Conclusion: Differences were observed between the two groups; however, a high loss to follow-up rate weakens the findings. Cervical expansive laminoplasty with preservation of unilateral PMLC may have the advantage of a lower rate of postoperative AS and maintenance of cervical spinal lordosis compared with the HSAP group.

\section{STUDY RATIONALE}

Postoperative AS are well described major and long-term complications of cervical laminoplasty for CSM. The overall incidence ranges from $6 \%$ to $60 \%$ [1-2]. The incidence and severity of AS appears to vary among different types of laminoplasty procedures. Further, various procedural approaches to the cervical spine may affect alignment, influence quality of decompression, as well as impair stability of the PMLC postoperatively [2-5].

\section{OBJECTIVE}

To determine if there is a difference in; (1) the rate and severity of postoperative AS; (2) the change of cervical spine sagittal alignment; and (3) spinal cord decompression between patients treated with a preservation of the unilateral PMLC procedure and HSAP for the treatment of CSM.

\section{METHODS}

Study design: Retrospective cohort study.

Inclusion criteria: We included all patients with CSM and presence of the following clinical conditions:

- three or more segments of spinal-cord compression

- developmental and degenerative stenosis of the cervical spine $+/$ - ossified posterior longitudinal ligament

- age 30-75 years

- presence of both preoperative and postoperative clinical and radiological examinations including $\mathrm{x}$ ray and magnetic resonance imaging (MRI) scans.

Exclusion criteria: Patients were excluded if they had:

- previous cervical spine surgery

- ankylosing spondylitis

- congenital deformities

- severe osteoporosis

- cerebrovascular disease sequela

- motor neuron disease, or neuromuscular junction disease

- required simultaneous instrumented fusion or combined anterior procedures

Patient population: Nine hundred and forty-six patients underwent one of two cervical laminoplasty procedures between January 2006 and December 2009. Of these, 394 (41\%) did not meet study criteria. There were 552 patients eligible for the study; however, 203 (37\%) did not have either radiology or clinical details in the follow-up records and 213 $(39 \%)$ did not return for follow-up. The remaining 136 of those eligible (24\%) were analyzed (Fig 1).

Interventions: Seventy patients received preservation of unilateral PMLC procedures (Fig 2) and 66 received HSAP" (Fig 3). The decompression segments were distributed from $\mathrm{C} 2$ to $\mathrm{T} 1$. The procedure was performed according to each surgeon's preference. There was no reason to believe that one procedure was selected over another based on disease severity.

Outcomes: The existence and severity of AS were measured and graded at final follow-up according to Kawaguchi's description [6] and Hosono grading system [7] by one of the investigators:

- No AS: not any axial symptoms at all

- Minor AS: slight symptoms which do not affect activities of daily living 
- Major AS: obvious symptoms and occasional analgesic pain medication may be required

- Severe AS: very obvious symptoms that affect patient's activities of daily living, regular analgesics are taken. The change of sagittal cervical spinal alignment was measured using the Ishihara [8] method by one of the investigators.

The expansive effect was evaluated by measuring the diameter of spinal canal on standard lateral view x-rays. A description of these methods can be found in the web appendices at www.aospine.org/ebsj.

Spinal cord decompression was measured using MRI, when available, by evaluating the appearance of the dura sac and the paraspinal muscles.

\section{Analysis:}

- Categorical baseline and outcome variables were compared using a Pearson Chi-square test.

- Continuous baseline variables, between group changes in preoperative to postoperative changes in cervical spine curvature and preoperative to postoperative changes in the expansive effect were compared using a 2-tailed independent $t$-test.

- Within group changes in preoperative to postoperative changes in cervical spine curvature and preoperative to postoperative changes in the expansive effect were compared using a 2-tailed matched pair $t$-test.

- We defined statistical significance as $P<.05$. Statistical analysis was performed using SPSS 16.0 software package.

Fig 1 Patient sampling and selection

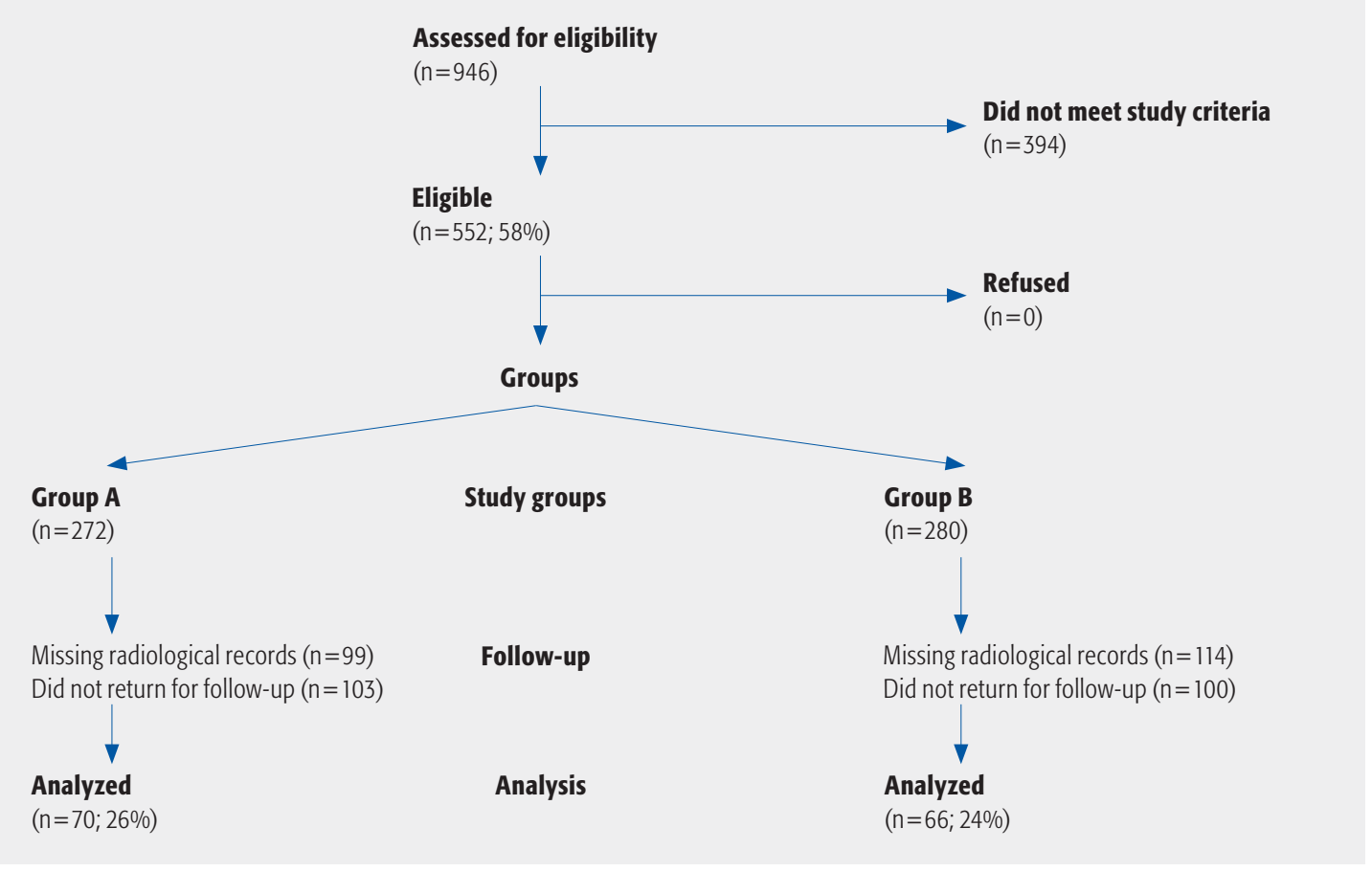

Fig 2 Procedure A: preservation of muscular-ligament complex method

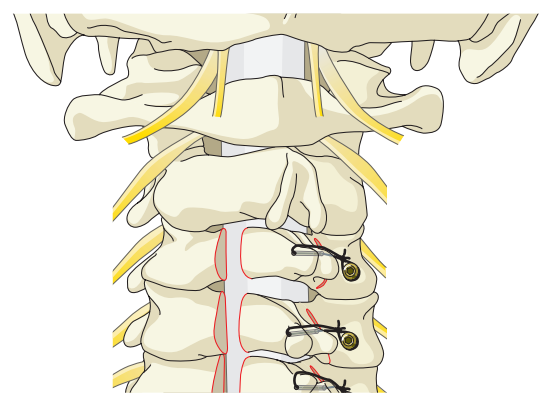

Figs 3a-b Procedure B: hinge side-anchoring method
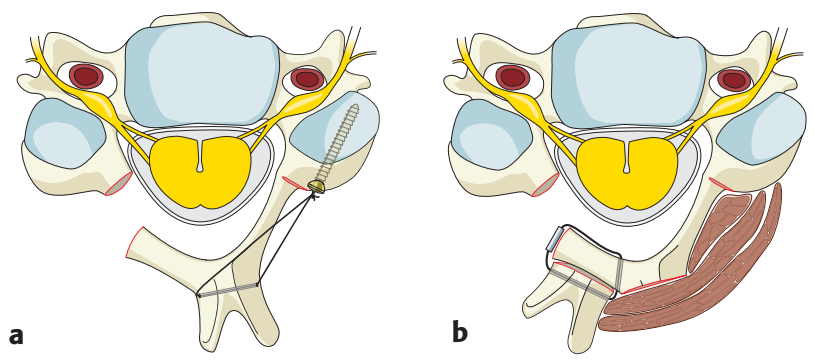


\section{RESULTS}

- Seventy patients in the preservation of unilateral PMLC group and 66 in the HSAP group were analyzed (Fig 1).

- The mean time to follow-up in the preservation of unilateral PMLC group was 6.9 months (range, 6-8 months) and the HSAP group was 6.4 months (6-8 months).

- There were no statistically or clinically significant differences between the two groups with respect to baseline demographic and preoperative Japanese Orthopaedic Association (JOA) scores (Table 1).

- There was no significant difference in intraoperative and immediate postoperative characteristics, such as operative time, blood loss between two groups, and postoperative JOA scores and recovery rates (Table 1). The most common decompression levels were C3-C7 for both groups, this difference was not statistically significant $(P=.23)$. Differences in other decompression levels were not clinically significant.
- The overall rate of any AS among all subjects was $35 \%$.

- No AS was reported in $45.7 \%$ of the PMLC group and $23.8 \%$ of HSAP $(P=.008)$ (Table 2). Severe AS was reported in $4.3 \%$ and $11.1 \%$ of patients per group, respectively $(P=.14)$ (Table 2).

- Cervical lordosis increased in the preservation of unilateral PMLC group and decreased in the HSAP group $(P=.04)$ (Table 3). The risk of losing lordosis was more than two times higher in the HSAP cohort compared with the PMLC group $(70 \%$ and $34 \%$, respectively; $P<.001$ ) (Table 4).

- All cases had satisfactory expansion of spinal canal (Table 5).

- There was a clear structure of muscular ligament complex without significant atrophy and fibrosis found in all preservation of unilateral PMLC cases. There was derangement and atrophy/fibrosis of the posterior muscular structure on the other side in all unilateral PMLC cases and both sides in all HSAP cases.

Table 1 Demographic and baseline characteristics of intervention groups

\begin{tabular}{llll}
\hline & $\begin{array}{l}\text { Group } \mathbf{A} \\
\mathbf{n = 7 0}\end{array}$ & $\begin{array}{l}\text { Group B } \\
\mathbf{n = 6 6}\end{array}$ & P-value \\
\hline Age in years at surgery (mean \pm SD) & $59.0 \pm 7.4$ & $57.2 \pm 8.3$ & .42 \\
\hline Female, $\mathrm{n}(\%)$ & $24(34.3)$ & $21(31.8)$ & .57 \\
\hline Standard follow-up, months (range) & $6.9(6-8)$ & $6.4(6-8)$ & .45 \\
\hline Preoperative JOA score $(\bar{x} \pm s)$ & $11.23 \pm 2.69$ & $11.15 \pm 2.70$ & .83 \\
\hline Postoperative and preoperative JOA score $(\bar{x} \pm S)$ & $2.35 \pm 2.30$ & $2.55 \pm 2.24$ & .631 \\
\hline JOA recovery rate, $\%$ & 42.1 & 46.7 & .39 \\
\hline Blood loss, $\mathrm{mL}$ & $234.3 \pm 113.2$ & $232.2 \pm 117.2$ & .138 \\
\hline Operating time, min & $92.8 \pm 18.8$ & $94.0 \pm 23.9$ & .748 \\
\hline Decompression level C3-C7, $\mathrm{n}(\%)$ & $56(80)$ & $47(71.2)$ & .23 \\
\hline
\end{tabular}

JOA = Japanese Orthopaedic Association 
Table 2 Incidence and severity of postoperative axial symptoms (AS) in two groups

\begin{tabular}{lllll}
\hline $\begin{array}{l}\text { Group } \\
\text { (No. of cases) }\end{array}$ & \multicolumn{2}{l}{ Number of cases (\%) } & & Major AS \\
\cline { 2 - 5 } & None of AS & Minor AS & $16(22.9)$ & $3(4.3)$ \\
\hline $\mathrm{A}(70)$ & $32(45.7)$ & $19(27.1)$ & $18(28.6)$ & $7(11.1)$ \\
\hline $\mathrm{B}(63)$ & $15(23.8)$ & $23(36.5)$ & & Severe AS \\
\hline
\end{tabular}

Fisher analysis $P=.048$

Table 3 The change of cervical spine curvature after surgery $(\bar{x} \pm s)$

\begin{tabular}{|c|c|c|c|c|c|c|}
\hline Group & $\mathbf{n}$ & Preoperative $\left({ }^{\circ}\right)$ & Postoperative $\left({ }^{\circ}\right)$ & Postoperative-preoperative $\left({ }^{\circ}\right)$ & $\begin{array}{l}\text { Within subject } \\
\text { P-value }^{*}\end{array}$ & $\begin{array}{l}\text { Between group } \\
\text { P-value }^{\dagger}\end{array}$ \\
\hline A & 70 & $12.28 \pm 10.93$ & $13.31 \pm 12.30$ & $1.03 \pm 7.99$ & & .04 \\
\hline B & 64 & $11.94 \pm 9.34$ & $10.31 \pm 10.07$ & $-1.63 \pm 6.45$ & & \\
\hline
\end{tabular}

${ }^{*}$ Matched pairs $t$-test

${ }^{\dagger}$ Independent $t$-test

Table 4 The maintenance and loss of cervical spinal lordosis after surgery

\begin{tabular}{lllll}
\hline Group & $\mathbf{n}$ & Maintained (\%) & Lost (\%) & $\boldsymbol{P}_{\text {-value* }}$ \\
\hline A & 70 & $46(65.7)$ & $24(34.3)$ & $<.001$ \\
\hline B & 64 & $19(29.7)$ & $45(70.3)$ & \\
\hline
\end{tabular}

*Pearson $\chi^{2}$ test.

Table 5 The expansive effect of cervical spinal canal after surgery $(\bar{x} \pm s)$

\begin{tabular}{|c|c|c|c|c|c|c|c|c|c|}
\hline \multirow[b]{2}{*}{ Level } & \multicolumn{4}{|c|}{ Group A (mm) } & \multicolumn{5}{|c|}{ Group B (mm) } \\
\hline & n & Preop & Postop & Postop-preop & n & Preop & Postop & Postop -preop & $\begin{array}{l}\text { Between group } \\
\boldsymbol{P} \text {-value* }\end{array}$ \\
\hline $\mathrm{C} 2$ & 8 & $16.4 \pm 1.4$ & $24.4 \pm 2.9$ & $8.0 \pm 2.5$ & 15 & $16.1 \pm 1.0$ & $20.3 \pm 1.6$ & $4.2 \pm 1.4$ & .000 \\
\hline $\mathrm{C} 3$ & 67 & $14.7 \pm 1.2$ & $21.4 \pm 2.7$ & $6.7 \pm 2.5$ & 62 & $14.8 \pm 1.3$ & $20.3 \pm 2.0$ & $5.5 \pm 2.0$ & .003 \\
\hline C4 & 70 & $14.2 \pm 1.3$ & $21.5 \pm 2.1$ & $7.3 \pm 2.0$ & 63 & $14.4 \pm 1.1$ & $20.9 \pm 2.0$ & $6.5 \pm 1.8$ & .014 \\
\hline C5 & 70 & $14.7 \pm 1.4$ & $22.2 \pm 2.3$ & $7.5 \pm 2.3$ & 63 & $14.4 \pm 1.2$ & $21.2 \pm 2.1$ & $6.8 \pm 2.1$ & .006 \\
\hline C6 & 69 & $15.3 \pm 1.6$ & $23.4 \pm 2.8$ & $8.1 \pm 3.1$ & 63 & $14.9 \pm 1.4$ & $22.4 \pm 4.7$ & $7.5 \pm 4.3$ & .132 \\
\hline $\mathrm{C7}$ & 61 & $15.7 \pm 1.9$ & $24.5 \pm 2.2$ & $8.8 \pm 2.8$ & 60 & $15.0 \pm 1.9$ & $21.8 \pm 2.4$ & $6.8 \pm 2.7$ & .000 \\
\hline Mean & & $14.9 \pm 1.6$ & $22.6 \pm 2$ & $7.7 \pm 2.6$ & & $14.8 \pm 1.4$ & $21.3 \pm 2.9$ & $6.5 \pm 2.8$ & .000 \\
\hline $\begin{array}{l}\text { Within su } \\
P \text {-value }\end{array}$ & & & .000 & & & & .000 & & \\
\hline
\end{tabular}

*Independent $t$-test

${ }^{\dagger}$ Matched-paired $t$-test 


\section{DISCUSSION}

- The rate of postoperative AS in this study is lower than most rates reported in the literature.

- The HSAP group presented higher rates and severity of postoperative AS, deterioration of cervical-spine lordosis, and worse derangement of paraspinal muscles.

- The preservation of unilateral PMLC procedure appears to have the advantage of less soft-tissue denervation that provides higher dynamic and static stability of the C-spine [9], better muscular alignment and power, and the possibility and capacity of early mobilization. These may contribute to the reduction of postoperative AS.

- Strengths: This is the first comparative study of two laminoplasty procedures evaluating important clinical outcomes. The patients appeared evenly matched with respect to the baseline measures that we collected except for the decompression levels between C2-7.

- Limitations: The greatest limitation of this study is the significant loss to follow-up $(76 \%)$ due to missing radiological materials or clinical details or nonreturning patients. Loss to follow-up can lead to selection bias. Although the losses were evenly distributed between the two groups, we cannot be sure that those lost were similar to those remaining; hence we cannot rule out bias in this study. Outcomes were not blinded or assessed by an independent investigator. Important factors that may also influence the outcomes that we cannot be sure were evenly distributed between the remaining subject groups include baseline symptoms severity, general health, smoking status, collar protection duration, and physiotherapy status.

\section{SUMMARY AND CONCLUSION}

Differences were observed between the two groups; however, a high loss to follow-up rate weakens our confidence in the findings. Laminoplasty with preservation of unilateral PMLC may have the advantage of a lower rate of postoperative AS and a satisfactory maintenance of cervical-spine lordosis compared with HSAP.

\section{REFERENCES}

1. Kawaguchi $\mathbf{Y}$, Kanamori $\mathbf{M}$, Ishihara H (2003) Minimum 10-year followup after en bloc cervical laminoplasty. Clin Orthop Relat Res; 411:129-139.

2. Ratliff JK, Cooper PR (2003) Cervical laminoplasty: a critical review. J Neurosurg; 98(3 Suppl):230-238.

3. Suk KS, Kim TK, Lee JH (2007) Sagittal alignment of the cervical spine after the laminoplasty. Spine; 32(23):E656-E660.

4. Shiraishi T (2002) A new technique for exposure of the cervical spine laminae: technical note. J Neurosurg; 96(1 Suppl):122-126.

5. Tani S, Isoshima A, Nagashima $Y$, et al (2002) Laminoplasty with preservation of posterior cervical elements: surgical technique. Neurosurgery; 50(1):97-101; discussion 101-102.

6. Kawaguchi $\mathbf{Y}$, Matsui $H$, Ishihara $H$, et al (1999) Axial symptoms after en bloc cervical laminoplasty. J Spinal Disord; 12(5):392-395.

7. Hosono N, Sakaura H, Mukai Y, et al (2006) C3-6 laminoplasty takes over C3-7 laminoplasty with significantly lower incidence of axial neck pain. Eur Spine J; 15(9):1375-1379.

8. Ishihara A (1968) Roentgenological investigation on the cervical lordosis of normal subjects. J Jpn Orthop Assoc; 42:1033-1044.

9. Nolan JP, Sherk HH (1988) Biomechanical evaluation of the extensor musculature of the cervical spine. Spine; 13(1):9-11. 


\section{EDITORIAL STAFF PERSPECTIVE}

This is our first scientific article published through the AOSpine methodological support offered through EBSJ in an effort to encourage a wider group of members from our global spine community to engage in regular clinical science undertakings. This article underscores some of the potential of providing methodological support to the regions; it also demonstrates the long road ahead in terms of overcoming infrastructural shortcomings in some of the regions.

The topic at hand was chosen for our EBSJ "enhanced methodological support program (EMSP)" after a fair bit of discussion among our editorial staff due to the relevance of the topic, the substantial number of patients studied, and the presence of two compelling comparison groups. The authors deserve praise for having diligently worked with the EBSJ methodological staff to optimize analysis of the available data points.

Substantial shortcomings, which could not be overcome, remain; however, the substance of the paper and aspects of the findings deserve publication and will provide substance for discussion. Hopefully a learning benefit will be gained for our scientifically minded AOSpine international community through increased methodological awareness, which not only apply to research but also to our clinical practices. Specifically the subject of loss to follow-up in retrospective studies is very common and of such relevance to the interpretation of data that we have dedicated a separate "Science in spine" section to this topic in the next edition of EBSJ. To be taken seriously as spine surgeons we need to try to optimize followup of our patients, not just for research but in our everyday practices. Of course there are tremendous financial and infrastructural hurdles to contend with. However, creative use of adjuvant strategies, such as structured telephone interviews, mailings of outcomes questionnaires, and use of regional providers notes with inclusion of the findings in a designated location of the available medical records system is a very acceptable fall-back option.

The subject of laminoplasty is an interesting and important topic of investigation. The occurrence of axial neck pain after laminoplasty is well known and is often taken into account when considering surgical options. The authors of this study have clearly presented their study objectives and their methodology. The figures provided also give the reader a clear understanding of the differences between the two procedures. Here are some noteworthy observations about this study:

- Although the study is well presented within the parameters of the journal, its largest weakness is the loss of follow-up for $76 \%$ of their patients, which the authors acknowledge. Despite demographic similarities, $24 \%$ of the eligible patient population is not a sufficient representation.

- In examining postoperative axial neck pain symptoms, it would be useful to examine the prevalence of preoperative axial neck pain symptoms. In the hinge group, if there were a disproportionate number of patients who had preoperative neck pain that did not change, or actually improved to "mild pain" after surgery, this may alter the final conclusions of the authors.

- In the discussion of pain having fixed time points of observation, ie, 2 years after elective procedures, is recommended to allow for a more consistent comparison of this outcomes variable.

- In general, it is not appropriate to declare that "there was no statistically significant difference," unless a prestudy power analysis had been performed and adequate power had been achieved by the study. It is more accurate to state, "We did not observe a statistically significant difference..."

- While the primary outcome of measure was the postoperative axial symptoms, it would be beneficial for surgeons to also know rates of complications, revisions, and failures of these procedures to have a wider scope of comparison. Since preservation of neurological status and, hopefully, improvement of myelopathy are main factors in performing laminoplasties, reporting of the neurological outcomes can be considered of prime outcomes parameters to study and report.

In the end the authors deserve praise for their effort in comparing outcomes. We hope that this presented project was helpful for the authors in implementing principles of clinical research for the future and will provide positive insights for our readership as well as in terms of methodological principles. We encourage your comments on the EBSJ "enhanced methodological support program (EMSP)." 